This essay will be submitted soon to Biology and Philosophy

\title{
On Griffiths and Gray's concept of expanded and diffused inheritance
}

\author{
Francesca Merlin \\ IHPST, University Paris 1 Panthéon-Sorbonne
}

francesca.merlin@gmail.com 


\begin{abstract}
$\underline{\text { Abstract }}$
Developmental System Theory is a theoretical reinterpretation of biological phenomena challenging the conventional gene-centered account of development and evolution. In this paper, I focus on Griffiths and Gray's version of Developmental Systems Theory and I particularly analyze their reconceptualization of inheritance. First, I present their concept of expanded and diffused inheritance; then, I examine and criticize their refusal of the multiple inheritance system model; finally, I present and contrast Griffiths and Gray's extension of what they call the "causal parity thesis" from development to evolution. I argue that their proposal is an interesting and programmatic philosophical perspective on biological phenomena but, because of their commitment to holism, fails to provide both more heuristic tools for empirical investigation in biology and a more realistic representation of the biological world.
\end{abstract}




\section{$\underline{\text { Introduction }}$}

Developmental System Theory (DST) is a label used by Griffiths and Gray (1994) to designate a reinterpretation of biological phenomena rejecting all dichotomous accounts of development and evolution ${ }^{1}$. More precisely, proponents of Developmental Systems Theory challenge the conventional gene-centric version of the Synthetic theory of evolution, according to which only genes, among determinants of phenotype, play a privileged causal role in evolution and carry the program for development, because it is "centered on a dichotomy between genes on the one hand and every other causal factor on the other" (Griffiths and Gray 2001: 197).

Griffiths and Gray' see their contribution to such a "theoretical reworking of biological concepts” (Oyama, Griffiths, Gray 2001: 1) as an improvement of Oyama’s general reinterpretation of development and evolution (Oyama 1985): their aim is to "confront one major weakness of previous presentations of the developmental systems idea - the lack of any way of delimiting and individuating developmental systems" (Griffiths and Gray 1994: 278). In line with the developmental systems tradition, Griffiths and Gray propose to discard the conventional account of development and evolution because it tends to distinguish resources into two fundamentally different kinds (e.g. genes-environment, nature-nurture, biology-

\footnotetext{
${ }^{1}$ Griffiths and Gray (1994: 278) claim that "Many authors have contributed to the developmental systems, or constructionist, tradition in the study of development. We have drawn on this tradition, and particularly on the work of Susan Oyama, to produce a general account of development and evolution". Oyama, Griffiths and Gray (2001: 2) also stress that "Developmental systems theory is not attributable to one person or group. It draws on insights from researchers in a wide range of area who have been dissatisfied with crude dichotomous accounts of development and have attempted to formulate an alternative". For more details about researchers belonging to DST or influencing it, see Oyama, Griffiths and Gray (2001: 9) and Griffiths and Gray (1994: 278, note 1).
} 
culture); rather, they suggest doing biology without such dichotomies. They do not simply propose new definitions for some central concepts in biology; they conceive a new general perspective on development, inheritance and evolution, and advance it as the basis for a new biological theory. Such a theoretical framework (DST) stresses the interactive and systemic character of all aspects of biological reality: according to Griffiths and Gray, it is a novel and more realistic representation of biological phenomena, and it can inspire new powerful paths in experimental research ${ }^{2}$.

In this paper, I focus on Griffiths and Gray's version of Developmental Systems Theory; in particular, I examine their proposal to redefine inheritance and its effects on the theory of evolution. Since inheritance can be considered as the interface between development and evolution, its reconcepualization seems to me a privileged point of view in order to understand in which sense and to what extent Griffiths and Gray's theoretical perspective for biology moves away from the Synthetic theory of evolution.

My primary aim is to show that, even though Griffiths and Gray's proposal represents an interesting and programmatic attempt to advance a new philosophical perspective on biological phenomena, they fail both to provide new heuristic tools for the empirical study of

\footnotetext{
${ }^{2}$ Oyama, Griffiths and Gray's (2001: 1-2) claim that Developmental Systems Theory "is not a theory in the sense of a specific model that produces predictions to be tested against rival models. Instead, it is $[\ldots]$ a framework both for conducting scientific research and for understanding the broader significance of research findings". Nevertheless, Griffiths and Gray $(1992,1994,1997,1998,2001,2004,2005)$ seem to suggest more than that (anyway, more than what Oyama (2000a, 2000b) suggests, i.e., a Developmental Systems Perspective or Approach, rather than Theory) because they repeatedly use the label "Developmental Systems Theory" and because, as I have just said, they stress that their reconceptualization both can provide alternative research programs for experimental investigation and more realistic theoretical models to represent biological phenomena.
} 
inheritance and evolution ${ }^{3}$ and to better grasp and represent biological phenomena, because of their commitment to holism (loosely speaking, the idea that, since everything causally depends on everything else, we cannot understand something without understanding everything $)^{4}$. By these terms, I do not want to claim that Griffiths and Gray's criticisms against the conventional version of the Synthetic Theory of evolution are uninteresting. Rather, I suggest that, by refusing important distinctions and differences among hereditary and evolutionary factors, Griffiths and Gray weaken the main potential of their criticisms against some dogmatic aspects of the traditional paradigm.

In the first part of this paper, I present the main features of Griffiths and Gray's redefinition of the concept of inheritance. In the second part, I particularly examine and criticize Griffiths and Gray's refusal to distinguish multiple systems, or channels, of inheritance in the name of their "constructive interactionism". In contrast with their ideas, I show the value of such a distinction as a heuristic tool for experimental research and as a more realistic representation of the phenomena of inheritance and evolution. In the third and final part, I present and criticize Griffiths and Gray's extension of what they call the "causal parity" thesis (or, "causal democracy", Oyama 2000b) from development to the case of evolution. I show that their radical refusal of dichotomies, in particular between genes and all

\footnotetext{
${ }^{3}$ In particular, if we compare Griffiths and Gray's proposal with other conceptions of extended inheritance (e.g. Sterelny et al's “extended replicator" model, 1996; Laland, Odling-Smee, Feldman's works on niche construction or ecological inheritance; Jablonka and Lamb's distinction of four dimensions or systems of inheritance, 2001, 2005).

${ }^{4}$ Different criticisms against the holistic character of Griffiths and Gray's version of Developmental Systems Theory are developed in Sterelny et al (1996), Kitcher (2001), Griesemer et al (2005) et Waters (2006).
} 
the other extra-genetic factors, leads them to drop some important evolutionary differences among hereditary factors. ${ }^{5}$

\section{I) Griffiths and Gray's reformulation of the concept of inheritance}

Griffiths and Gray claim that the traditional concept of inheritance needs "substantial reformulation" because it is based on dichotomies between genes and other developmental factors which are not grounded on empirical differences, but on "the metaphysical distinction between 'form' and 'matter' " (Griffiths and Gray 2004: 415, Griffiths and Knight 1998). Let us consider in details their proposal.

First, they reformulate the concept of inheritance in opposition to the traditional population genetics' conception of evolution as change in gene frequency: they oppose the idea that inheritance is the passing on and changing of master molecules (genes, or DNA sequences) considered as the only carriers of information for development, and so the only factors playing a causal role in evolution. Their privileged target is Dawkins' interpretation of the theory of evolution, namely the idea of "extended phenotype": "The facts of evolution do not justify a special role for DNA sequences as 'replicators' whilst membranes are lumped together with every thing from methyl groups to human culture as 'interactors"” (Griffiths and Knight 1998: 254). The second reason leading Griffiths and Gray to reformulate the concept of inheritance is the absence of developmental considerations from the Synthetic theory of evolution: according to them, by reintroducing development into the evolutionary theory through a reconcepualization of inheritance, biologists should be able to pay the appropriate

\footnotetext{
${ }^{5}$ As I said above, the core of my criticisms precisely concerns Griffiths and Gray's version of Developmental Systems Theory. Nevertheless, I will also refer to some papers by Oyama in order to better clarify Griffiths and Gray's perspective.
} 
attention to a variety of factors playing equally important roles in development and in evolution.

On these bases, Griffiths and Gray propose a "principled" definition of inheritance justified by the meta-theoretical claim that "the concept of inheritance is used to explain the stability of biological form from one generation to the next" (Griffiths and Gray 2001: 196) ${ }^{6}$. Thus, according to them, inheritance is "the reliable reproduction of developmental resources down the lineage" (Griffiths and Gray 2001: 214) and this concept applies to "any resource that is reliably present in successive generations, and is part of the explanation of why each generation resembles the last" (Griffiths and Gray 2001: 196).

First of all, it is important to clarify that Griffiths and Gray do not use the expression "reproduction of developmental resources" according to its standard meaning, i.e. by referring to the replication of hereditary factors and their material transmission from parents to offspring; rather, by the term "reproduction" they mean the reappearance or the reconstruction of developmental resources in successive generations. Actually, one of the most important novelties of Griffiths and Gray's reconcepualization of inheritance is the refusal of the notion of transmission and its substitution with the notion of stability. More precisely, Griffiths and Gray move away from the idea of "hereditary transmission of traits or coded representations of traits" (i.e., genes), and even completely "relinquish the conviction that traits can be transmitted at all" (Oyama 2000a: 6) because, according to them, the notion of transmission is deeply-rooted in the dualistic tradition of the Synthetic theory assuming the dichotomies between nature and nurture, organism and environment, development and evolution. They refuse the distinction between, on the one hand, a blueprint (i.e., nature) considered as the only factor passed on from parents to offspring and the privileged carrier of a program for

\footnotetext{
${ }^{6}$ Griffiths and Gray's concept of expanded inheritance "in principle" fits in with the goal of the traditional notion of inheritance: both are used to explain the correlation of resemblance between parents and offspring.
} 
development and, on the other hand, a standard background of other inputs to development. They claim that such a distinction is a residual form of pre-formationism because it considers transmitted factors (e.g., genes) as containing all the relevant information to construct offspring like their parents. Furthermore, they also radically reject extending the use of the notion of transmission to extra-genetic hereditary factors because transmission is simply a metaphor implying a denial of development (Oyama 2000a: 70).

For these reasons, Griffiths and Gray defy the traditional dichotomy-based perspective on inheritance, development and evolution, and argue that "nature" is not genotypic and transmitted, but phenotypic and constructed at each generation through developmental interactions they call "nurture"7 . By resolving the nature-nurture dichotomy in this way, Griffiths and Gray intend to do justice to the facts of development and to assess the evolutionary potential of various forms of inheritance. They move to a concept of expanded inheritance by replacing the notion of transmission with the notion of stability of developmental resources and interactions in successive generations. More precisely, they use the term "stability" to mean reliable presence, reoccurrence, reconstruction, of developmental resources and interactions at each generation: "developmental means are transmitted in the sense of being available during reproduction and ontogeny" (Oyama 2000a).

So, what does an organism inherit according to Griffiths and Gray's definition of inheritance? Much more than just nuclear DNA! More precisely, their definition entails a radical expansion of the concept of inheritance which turns out to apply to the following genetic and extra-genetic developmental resources: environmental-physical resources persisting independently from organisms (more precisely, what is inherited is the interaction

\footnotetext{
7 "All phenotypes are constructed, not transmitted [...] Not only are traits not directly transmitted across generations, nor are blueprints, potentials, programs or information for the traits. Instead, all phenotypes must develop through organism-environment transactions" (Gray 1992: 177).
} 
with persistent resources affecting the organisms, e.g., gravity for organisms of big size; Brownian motion for microorganisms); resources collectively generated by the members of a population (e.g., behavioral and social traditions, some features of the habitat like nests, dams, etc.); resources due to parents (e.g., genes, endosymbionts, chromatin marks, cytoplasm including organelles, membrane structures, antibodies and mithocondria, behaviors); resources self-generated during the developmental process (e.g., proteins produced by gene expression). All these developmental resources and their reciprocal interactions are inherited, i.e. "stable" from one generation to the next. According to Griffiths and Gray, they explain the stability of biological forms across generations and constitute what they call the "Developmental System" (DS): they represent the set of developmental resources and interactions (i.e., developmental factors) participating in the reconstruction of the developmental process, or life cycle, in each generation (Griffiths and Gray 1994: 285).

Even though the extension of the hereditary package to include more than nuclear DNA is actually recognized by biologists ${ }^{8}$, Griffiths and Gray’s proposal represents a further expansion of the concept of inheritance because, as I already said above, it applies not only to extra-genetic intracellular factors (Sterelny et al 1996, Jablonka and Lamb 2005) and to extragenetic factors due to the "niche construction" activity (Laland, Odling-Smee, Feldman 2003), but also to resources generated during the developmental process of organisms.

Moreover, Griffiths and Gray consider "processes" (interactions) as inherited developmental factors across generations. This is an important consequence of the theoretical perspective for biology they advance, which assigns a primary importance to the dynamic interaction and the context-dependence of all biological phenomena. Actually, they take it a step further rejecting the very distinction between organism and environment: in contrast with the traditional view, organism and environment "interpenetrate" (Oyama 2000a: 3) and form a

\footnotetext{
${ }^{8}$ However, extra-genetic inheritance has not yet been introduced into population genetics' models of evolution.
} 
dynamic and interactive organism-environment system (E) - i.e., the Developmental System (DS) - evolving as a whole.

It is also worth noting that, despite such an extension of the concept of inheritance, Griffiths and Gray do not particularly investigate the mechanism(s) by means of which the system of developmental resources and interactions (DS) reoccurs more or less reliably at each generation (i.e., is inherited over generations). They reject Dawkins' distinction between "replicators" and "vehicules" (or "interactors", in Hull's terms) which is grounded on "the supposed asymmetry between the role of genes and the role of other developmental resources" (Griffiths and Gray 1994: 299); moreover, they want to "evaporate" such an asymmetry and to completely stop talking in terms of "replicator" and "interactor" because such a dichotomy implies a privileged causal role in development and in evolution for some factors (replicators) over the others.

Griffiths and Gray also refuse the "extended replicator" perspective (Sterelny et al 1996) according to which genes are not the only replicators. However, in order to answer Sterelny et al's (1996) criticisms to Developmental Systems Theory and to criticize their distinction of a variety of replicators, Griffiths and Gray use the term "replicator" in claiming that "according to the developmental system theory, all developmental interactions are replicated as part of the replications of the developmental process/life cycle. [...]"; in fact, "if we insist that a replicator have the intrinsic causal power to replicate itself, there will be only one replicator, the life cycle".

In what does the developmental process" "intrinsic causal power to replicate itself" consist? First, Griffiths and Gray do not use the term "replicator" referring to special factors able to produce more or less reliable copies of themselves (Hull and Wilkins 2005); rather, by this term they designate the process of reconstruction, at each generation, of the developmental process through the interactions among inherited developmental factors. 
Second, they sometimes try to specify more precisely what kind of constructive process is in question in inheritance (i.e., how the developmental process reconstructs itself in each generation) by claiming that "the process is self-organizing" (Gray 1992: 181): "speciestypical traits are construed by a structured set of species-typical developmental resources in a self-organizing process" (Griffiths and Gray 1994: 181). Nevertheless, Griffiths and Gray fail in providing an explanation of what they mean by "self-organization" because they do not introduce any analytic physico-chemical description of how such a biological mechanism should work 9 .

\section{II) Griffiths and Gray's concept of diffused inheritance: how to deal with it}

\section{in biology?}

In this second part, let us examine Griffiths and Gray's refusal to distinguish multiple systems, or channels, of inheritance in interaction ${ }^{10}$. Griffiths and Gray do not simply reject the traditional idea that inheritance is localized in some genetic factors; they also refuse any particular or privileged localization of inheritance and, by contrast, they propose a concept of diffused inheritance ${ }^{11}$ according to which organisms do not inherit many factors but the entire

\footnotetext{
${ }^{9}$ This point is further discussed in Barberousse (this issue).

${ }^{10}$ Griffiths and Gray particularly refuse the idea that the interactions among systems of inheritance are mere additive. Anyway, they radically move away from the idea that we could distinguish different systems of inheritance, even if their interactions are considered as non-additive (non-linear). They claim that one of the main motivations to refuse the distinction of multiple systems in interaction is "to draw attention to fact that developmental causes do not have their effects in isolation, but as part of a wider system of causes". Therefore, "the very idea of separate systems" is inadequate because it "suggests autonomy" (Griffiths and Gray 2001: 197198). According to Griffiths and Gray, Developmental Systems Theory provides a new conceptual framework that avoids this.

${ }^{11}$ Or, “a ‘systemsy’ notion of heredity”, Griesemer et al 2005: 524.
} 
developmental matrix (i.e., the system of resources and interactions participating to the reconstruction of the developmental process). In this manner, they want to stress the intertwined relation and the interdependence among genetic and extra-genetic hereditary developmental factors, not only in the developmental process, but also in inheritance across generations: they claim that it is "more biologically realistic and, in the long run, more productive to think of the life cycle being reconstructed by a system of resources" (Griffiths and Gray 2001: 196).

In opposition to Griffiths and Gray's “constructive interactionism”, I argue that, even if we adopt the idea that what is inherited is not a set of distinct factors but a whole system of non-linear (i.e., non-additive) interactions among developmental factors, it is heuristically and realistically important ${ }^{12}$ to distinguish some sub-systems of inheritance on the basis of what we could call their "quasi-independence": such sub-systems should be identified by the interactions among their respective own elements, which would be stronger than the interactions among elements belonging to different sub-systems. Anyway, to say that different sub-systems of inheritance are quasi-independent from each other does not mean that they could subsist and function in complete isolation from each other. For instance, genes could not replicate and pass on from parents to offspring, and could not participate to the developmental process, if they were not surrounded and supported by all the other nongenetic developmental factors. The quasi-independence of sub-systems of inheritance allows us to describe, analyze and explain them independently from the others; however, their

\footnotetext{
${ }^{12}$ I use the terms "heuristic" and "realistic" respectively in relation to the methods used to solve a problem and to characterize the match of a real phenomenon and its representation (model).
} 
reciprocal interactions have to be considered too, in order to be integrated in the description, the analysis and the explanation of the whole system of inheritance. ${ }^{13}$

Biologists currently apply this kind of distinction in experimental and theoretical research. Through some examples, I intend to argue that the distinction of different systems of inheritance is not simply heuristically useful for empirical studies, but it is also important as realistic representations of biological inheritance: such a distinction allows biologists to better investigate, represent, and explain the relative independence of diverse aspects of inheritance and their reciprocal influences. Griffiths and Gray's claim that each developmental factor depends on the presence and functionality of the others seems to me trivially true and a weak basis to argue, as Griffiths and Gray do, against the importance of distinctions in experimental and theoretical biology.

In their later book (2005), Jablonka and Lamb distinguish four systems, or dimensions, of inheritance and analyze their reciprocal interactions. It is in virtue of such a distinction that they can proceed to analyze and explain the interplay among different systems of inheritance (their respective quasi-independence and their reciprocal influences). For instance, they examine the relationship between what they call the genetic system and a variety of epigenetic systems of inheritance ${ }^{14}$ (Jablonka and Lamb 2005: chapter 7). On the one hand, epigenetic

\footnotetext{
${ }^{13}$ Griesemer et al (2005: 526-528) provide a different argument in favor of the distinction of multiple systems of inheritance. First, they argue for the compatibility of multi-system models with the context-dependence of each system on the others; second, they stress the important difference between the separateness of some factors in interaction and their causal or statistical independence. Griesemer et al's argument turns out to partially support Griffiths and Gray's conception of inheritance because they conclude that the distinction of multiple systems of inheritance is compatible with the characterization of inheritance in a holistic way.

${ }^{14}$ Jablonka and Lamb (2005, chapter 4) distinguish different kinds of epigenetic systems of inheritance: the inheritance of patterns of gene activity (self-sustaining loops), the inheritance of cell structure, the chromatinmarking system of inheritance, the inheritance of silencing of specific genes by RNA interference.
} 
systems can have different types of effects on the genetic system: "epigenetic marks affect not only gene activity", they also can directly bias the generation of variation by affecting "the probability that the DNA region will undergo genetic change" (Jablonka and lamb 2005: 246250); epigenetic changes induced by changing environmental conditions can affect ("guide") the selection of variants by revealing previously hidden genetic variation (i.e., the process of genetic assimilation) (Jablonka and Lamb 2005: 258-276). On the other hand, the genetic system of inheritance can also influence epigenetic systems "by affecting the marks genes carry, the nucleotide sequences of the RNAs involved in RNA interference, the amino acid sequences of the proteins that form heritable cell structures or have a role in self-sustaining loops, etc". (Jablonka and Lamb 2005: 276).

Jablonka and Lamb also provide many examples showing the developmental and evolutionary significance of the interactions between what they call the genetic system and, respectively, the behavioral and the cultural systems of inheritance, and they examine how genetic, behavioral and cultural changes impinge on each other. They describe many cases of co-evolution of genes and culture characterized by feedbacks between inherited genes and the inherited niche (i.e., the Baldwin effect ${ }^{15}$ ). For instance, changes in human lifestyle can influence changes in gene frequency, as in the case of dairying, in which cultural evolution leading to milk drinking has influenced the frequency of the lactase-persistence allele ${ }^{16}$ (Jablonka and Lamb 2005: 292-296). Another similar example is the case of Tay-Sach disease: actually, there is evidence that Jewish habits, in particular the fact that they are often

\footnotetext{
${ }^{15}$ The Baldwin effect occurs when a biological trait becomes genetically fixed in a population as a result of first being learned.

${ }^{16}$ The lactose in milk has to be broken down into simple sugars in order to be easily absorbed into the bloodstream; this require the enzyme lactose which is coded by the lactase-persistence allele. See Durham 1991, who analyzes in the case of dairying how changes in human lifestyles have influenced the frequencies of some of their genes.
} 
forced to live in slums and ghettos, have influenced the frequency of the Tay-Sachs allele among Ashkenazy Jews ${ }^{17}$ (Jablonka and Lamb 2005: 297-298).

After the analysis of other cases in which the interactions and influences between the genetic and the cultural or the behavioral systems of inheritance play a relevant developmental and evolutionary role, Jablonka and Lamb conclude by claiming that: "The dynamics of the interactions between cultural and genetic changes is complex and difficult to unravel, and we made no attempt to describe them for either the milk or malaria stories. Common sense and lot of indirect evidence suggest that learned, socially transmitted behaviors occupy the driver's seat of coevolutionary change, simply because adaptation can occur much more rapidly through behavior than through genetic change" (Jablonka and Lamb 2005: 295). Thus, Jablonka and Lamb do not pretend to claim that there is evidence for the privileged role of one system of inheritance over the others in the evolutionary process; however, they simply show that such distinctions have a central value in the experimental and theoretical studies of inheritance because they reflect the quasi-independence of each system and, at the same time, they show the pathways of reciprocal influences among them (this point is in contrast with Griffiths and Gray's refusal of any distinction between systems of inheritance).

I argue that Jablonka and Lamb's analysis shows that it is worth separately considering different systems of inheritance not simply because, in this way, it is easier for biologists to experimentally study them (i.e., for reasons of heuristic usefulness); rather, their distinction has a biologically realistic value too, as it correctly represents and allows us to understand the very dynamics of inheritance going on in living organisms.

\footnotetext{
${ }^{17}$ Tuberculosis is rife in slums and ghettos and people with the Tay-Sach allele are less likely than other people to develop it.
} 
Another enlightening case study showing the importance of distinctions between systems (or quasi-independent units) of inheritance is the case of CRISPRs loci in Archaea and Bacteria (Makarova et al 2002, Godde and Bickerton 2006, Barrangou et al 2007). "CRISPRs" means Clustered Regularly Interspaced Short Palindromic Repeats. These DNA repeats, which are about $21-47$ base pairs in length and are interspersed with non-repetitive sequences of similar length, have been found in a wide range of diverse prokaryotes, including Archaeal and Eubacterial species. Moreover, a number of cas CRISPRs-associated genes have also been characterized in many of the same organisms. CRISPRs, together with the associated cas genes, seem to provide resistance against phages (the resistance specificity is determined by spacer-phage sequence similarity). Phylogenetic analysis suggests that the CRISPRs loci have been propagated via horizontal gene transfer among very distantly related genomes, and that "the gene-cassette disseminated as a single entity" (Godde et al 2006: 719). As I have just said above, CRISPRs-containing species of prokaryotes are extremely diverse, belonging to the domain Archaea as well as to the majority of the phyla that have been sequenced from the Eubacterial domain.

The CRISPRs case study seems to me a good example in support of the biologically realistic value of distinctions among different systems of inheritance, which continuously are in non-additive interaction but, at the same time, keep a relative autonomy (i.e., a quasiindependence) from each other. In fact, even if CRISPRs loci could not subsist in the genome of diverse Prokaryotes and could not move from one organism to another in the absence of all the other developmental systems or factors (other genes, epigenetic factors, environmental extra-genetic factors, etc.), they do not strictly depend on a specific set of genetic and extragenetic factors, but they can move as an isolated and autonomous unit and insert in the genome of very different organisms (Prokaryotes belonging to the Archaea and to the Eubacterial domains). In other words, CRISPRs are characterized by some autonomy with 
respect to the other genetic and extra-genetic hereditary factors as to their inheritance (i.e., transmission, or, in Griffiths and gray's term, stability) from one organism to another.

Taking the CRISPR case study as an example, I would challenge Griffiths and Gray to describe and explain such a phenomenon of horizontal inheritance in terms of what they call a "constructive interactionism" - i.e., how the DST framework, characterized by a systemic and interactive conception of inheritance and by the refusal of distinct systems of inheritance, could account for such a phenomenon of inheritance. It seems to me that Griffiths and Gray should be obliged to acknowledge that, for instance in the case of CRISPRs' horizontal inheritance, distinctions are not only useful, but they also realistically represent the relative quasi-independence of some hereditary factors from the others (e.g., the relative autonomy of CRISPRs' horizontal transfer). In more general terms, I question the advantage Griffiths and Gray's conception of a systemic, interactive, and diffused inheritance would provide in comparison with other approaches distinguishing different systems of inheritance in nonadditive interaction (e.g., the four systems of inheritance, Jablonka and Lamb 2005; the “extended replicator" model, Sterelny et al 1996).

As Gray says, "biologists often want to know the research implications of adopting a particular theoretical perspective (Gray 2001: 28). I also would ask about the research implications of adopting Griffiths and Gray's concept of expanded and diffused inheritance, i.e. what types of research it encourages and what advantages such a holistic approach would provide in comparison with other approaches already used in experimental biology. ${ }^{18}$

\footnotetext{
${ }^{18}$ It is interesting to notice that Griffiths and Gray's claims about the value of their perspective for biological research oscillate between two slightly different positions. Sometimes, they maintain that their holistic vision (their "constructive interactionism") provides both a more heuristic and realistic representation of inheritance and evolution than other approaches: "we have strong reservation about multiple inheritance or "extended replicator' models. We believe that it is both more biologically realistic and, in the long run, more productive to think of the life cycle being reconstructed by a system of resources" (Griffiths and Gray 2001: 196). Other times,
} 
Throughout their papers, Griffiths and Gray seem to defend the idea that the Developmental Systems Theory (DST) is a programmatic philosophical perspective which can be useful to rethink developmental and evolutionary models in theoretical biology ${ }^{19}$ and to re-orient research programs in experimental biology.

In Gray (2001) and in Griffiths and Gray (2001), they particularly maintain that "the benefits of this reconceptualization of evolution in Developmental Systems Theory terms are considerable", and they advance "some suggestions for the kind of research questions [...] Developmental Systems Theory encourages". It would promote the following research "tactics" about inheritance: the study of extra-genetic inheritance, in particular the investigation of whether there are adaptive mechanisms for passing on extra-genetic inheritance, the investigation of their effects, and the development of mathematical models for studying the impact of different types of extra-genetic inheritance and their co-evolution with genetic change; the study of niche construction, and the testing of predictions due to niche construction models by using comparative methods. I argue that, if these are the main points of the re-orientation Developmental Systems Theory provide in experimental studies about inheritance, Griffiths and Gray's "novel” perspective is not so new and does not involve any

they maintain that their perspective is more biologically realistic in order to think and represent the dynamics of inheritance and evolution but, at the same time, they acknowledge the heuristic value and the inevitability of distinctions in studying inheritance and evolution in particular cases: "A central theme of the Developmental Systems Theory research tradition has been that distinctions between classes of developmental resource should be fluid and justified by particular research interests, rather than built into the basic framework of biological thought $[\ldots]$ The developmental system is not two things, but one, albeit one the can be divided up in many ways for different theoretical purposes" (Griffiths and Gray 2004: 419-420).

${ }^{19}$ Griffiths and Gray's primary aim is to provide a philosophical program which can be translated by biologists into a new biological theory: so, they themselves do not explicitly put forward any new theoretical model to represent development and evolution. However, they claim that their perspective can provide the philosophical clarity to outline a new research program in biology. 
re-focalization of biological research. Actually, all the research questions suggested by Griffiths and Gray are already topics of investigation about inheritance in experimental and in theoretical biology and do not need a radical reworking of biological concepts in order to be considered by biologists $^{20}$. Furthermore, all these kinds of investigation can be performed only by distinguishing different systems (or factors) of inheritance, and by studying their relative independence and their reciprocal influences in particular cases - i.e., what Griffiths and Gray refuse from a theoretical point of view ${ }^{21}$.

Griffiths and Gray's holistic idea that organisms inherit the entire developmental matrix is certainly more rich and complete than alternative accounts of inheritance limited to a

${ }^{20}$ I guess that Griffith and Gray would reply to me by suggesting that biological researches focused on extra-
genetic inheritance provide the evidence for the empirical applicability of Developmental Systems Theory in the study of inheritance. More generally, Griffiths and Gray seem to consider almost the entire book Cycles of Contingency (2001) as a collection of biological works according to Developmental Systems Theory. On the contrary, I suggest that, even though these works focus on some major themes of Griffiths and Gray's version of Developmental Systems Theory (i.e., the role of development and of extra-genetic inheritance in evolution, the importance of interactions among developmental factors and between organism and environment), they cannot be considered as evidence for its empirical applicability in biological research because, contrary to Griffiths and Gray's version of Developmental Systems Theory, they do not characterize inheritance in a holistic way (cf. Jablonka and Lamb 2001 and Laland, Odling-Smee, Feldman 2001, in Cycles of Contingency).

${ }^{21}$ For instance, Kitcher (2001), even though sympathetic to the interactionism of Developmental Systems Theory, stresses that its empirical applicability in biology is a highly problematic task. In particular, he shows that Developmental Systems Theory cannot give an answer to the obvious question a sympathetic biologist reading Griffiths and Gray would ask, which is "How do I put these ideas to work in concrete situations?". Kitcher continues: "This does not mean that $[\ldots]$ 'developmental systems theory' should be abandoned but that this kinds of work needed to make them viable pieces of biological theory are specific models for tackling interesting problems (rather than philosophical diagnoses of previous errors). It would be very interesting, for example, to see a developmental systems analysis of early development in Drosophila [...] substituted for some part of population genetics" (Kitcher 2001: 413). 
small set of hereditary factors in additive interaction. Nevertheless, I argue that, if we understand inheritance as a whole system composed of multiple quasi-independent subsystems of inheritance in non-additive interaction (that is not Griffiths and Gray's representation of the inheritance system), it is more useful and more biologically realistic to maintain their relative distinctions in order to understand their interactions and reciprocal influences. Griffiths and Gray's holistic approach turns out to be less informative than the representation of inheritance I defend, because it simply tells us that organisms inherit a very complex system of non-linear interactions, but it says nothing more about the dynamics allowing such a system to be inherited (to re-occur or to be reconstructed in each generation, in Griffiths and Gray's terms).

For this reason, Griffiths and Gray's attempt to provide some new basis for research in theoretical and experimental biology seems to collapse: by adapting Oyama's words (Oyama 2000b: S343), I suggest that what they propose is quite a general revision of biological concepts which cannot pretend (at least in the case of the reconceptualization of inheritance) to provide a more realistic representation of the biological dynamics and more powerful tools for empirical investigations and predictions ${ }^{22}$. In other words, I do not deny the value of Griffiths and Gray's version of Developmental Systems Theory as an interesting philosophical perspective for looking at biological reality with different eyes (i.e., focusing on its systematic and interactive character). Nevertheless, when it is in question to translate their reconceptualization into new theoretical models and to apply it in empirical studies, its holistic character lets out to be problematic.

In particular, the application of Griffiths and Gray's proposal in experimental biological research reveals an impracticable task: it is impossible to analyze and explain how

\footnotetext{
${ }^{22}$ See note 2 about the contrast between what Oyama, Griffiths and Gray claim (2001) about Developmental Systems Theory as a theory, and what Griffiths and Gray maintain in their own papers.
} 
genetic and extra-genetic factors in interaction (i.e., "the entire developmental matrix") are passed on across generations and have an impact on the evolutionary process without analyzing, distinguishing and untangling all systems, or factors, of inheritance. I suggest that Developmental Systems Theory does not collapse into an "unmanageable holism" (Sterelny et al 1996) only because Griffiths and Gray are compelled to acknowledge one point they refuse from the philosophical point of view: they recognize that, in biology, experimental investigation without distinctions is an impossible task.

\section{III) No place for some important distinctions in the evolutionary "causal parity" thesis}

First of all, let us recall that Griffiths and Gray refuse the conventional definition of evolution as change in gene frequency because it attributes an evolutionary causal primacy to genes. In contrast, they propose to extend what they call the "causal parity" thesis from development to evolution: this means that, according to them, no hereditary factor has a privileged causal responsibility either in development or in evolution: even though they can have different causal roles, they are all on a par as to explain the evolutionary process.

Let us examine more in detail Griffiths and Gray's reasoning to argue for the "causal parity" thesis and for their reconceptualization of evolution. First, they claim that "organisms inherit much more than just DNA" (Gray 2001: 7), that is many other extra-genetic developmental resources (i.e., persistent resources, collectively generated resources, parental resources, and self-generated resources) and the interactions among them. Second, they suggest that, as an extra-genetic factor is inherited, it plays an evolutionary causal role. Third, from the evidence for extra-genetic inheritance and its causal role in evolution, they argue, against the conventional view, that all genetic and extra-genetic hereditary factors are "on a 
par" as to their causal responsibility in the evolutionary process: "the role of genes is not more unique than the role of many other factors $[\ldots]$ : the supported asymmetry between the role of genes and the role of other developmental resources evaporates when closely analyzed $[\ldots]$; other developmental resources do just the same" (Griffiths and Gray 1994: 277, 299). In other words, they argue that neither metaphysical nor empirical distinctions can be used in order to confer a causal primacy to one kind of hereditary factors (e.g., genetic factors) with respect to the others (e.g., extra-genetic factors) in evolution (this is what they call the "causal parity" thesis $)^{23}$.

Finally, on the basis of the "causal parity" thesis, they conclude that evolution cannot be defined as change in gene frequency; rather, "evolution can be narrated from a Developmental Systems perspective that does not privilege any component of the system" (Griffiths and Gray 2004: 420): it is "change over time in the developmental system (DS) of a lineage" (Griffiths and Gray 2004: 422) ${ }^{24}$. Thus, Griffiths and Gray consider the Developmental System both as the unit of development and evolution; as they explicitly claim, they think that "Evolutionary causes are not different from developmental causes"

\footnotetext{
${ }^{23}$ In particular, one way Griffiths and Gray argue in favor of the idea that all genetic and extra-genetic factors are on a par as regard their causal responsibility in development and in evolution (i.e., the "causal parity" thesis) is by criticizing the idea that only genes are information-carriers: according to them, no characterization of the concept of information can isolate genes as privileged cause of development and evolution because any definition of information which can apply to genes can also equally apply to all the other extra-genetic factors See Lorne's abstract in this volume.

${ }^{24}$ Griffiths and Gray propose a variety of slightly different definitions of evolution. Here is a sample: "Evolution is change over time in the composition of populations of developmental systems" (Griffiths and Gray 2001: 214); "Evolution is change in the nature of populations of developmental systems" (Griffiths and Gray 2004: 420); "Evolution is change across generations in the distribution and composition of populations of developmental systems" (Gray 1992: 182).
} 
(Gray 1992: 187): all hereditary resources and interactions have equally important causal roles both in development and in evolution.

I argue that Griffiths and Gray's argument for what they call the "causal parity" thesis is not sufficiently rigorous and warranted for the following reason: they do not provide any empirical evidence to support the claim that all genetic and extra-genetic hereditary factors are equally important as regard their evolutionary causal role and so are on a par as to explain evolution. On the contrary, they completely ignore that hereditary factors can differ in some important respects, in particular as to the scope of their effects across evolutionary time. Actually, I argue that the evolutionary potential of hereditary factors transmitted (stable, in Griffiths and Gray's terms) over many generations is more important than the evolutionary potential of hereditary factors transmitted over only one generation because the former, by being transmitted more or less reliably across many generations, can be subject to several events of selection and drift, and so can potentially lead up to more significant long-term evolutionary consequences (for instance, adaptations) than the former.

By this claim, I do not intend to deny the evolutionary causal role of hereditary factors transmitted over only one generation; on the contrary, I even suggest that factors not transmitted at all (i.e., in Griffiths and Gray's terms, not stable over successive generations) can have a causal responsibility in evolution as they can play a role in the differential survival and reproduction among organisms. Moreover, I do not even intend to defend the causal primacy of genes in evolution as conceived by the conventional view. Rather, I want to argue that there is a causal asymmetry, not between genes and all the extra-genetic factors, but between hereditary factors transmitted over many generations and hereditary factors transmitted over only one in terms of the scope of the evolutionary effects they can 
respectively produce ${ }^{25}$. In particular, in order to stress some limits of Griffiths and Gray's "causal parity" thesis, I intend to underline the two following points: first, for an extra-genetic developmental factor to be hereditary is not a sufficient condition to be on a par with genes as an evolutionary cause; second, for a factor to be hereditary is not even a necessary condition to have a causal role in evolution.

So, in contrast to Griffiths and Gray, I suggest to distinguish 1) developmental factors which are transmitted over only one generation (e.g., in the case of mammals, cytoplasm factors like intracellular chemical gradients and antibodies, methylation marks, extracellular chemical influences, etc.), and 2) developmental factors which are transmitted over many generations (e.g., in the case of mammals, genes, endosymbionts, mithocondria, some factors due to the activity of niche construction, etc.).

First, let us consider developmental factors transmitted over only one generation (e.g., from generation1 to generation2). Such factors explain the resemblance between parents (generation1) and offspring (generation2). They can contribute to the differential fitness among generation1-organims in a generation1-population: this means that they can have a causal responsibility in the differential survival and reproduction among generation1organisms, so they can be differentially passed on to generation2-organisms (offspring). However, factors transmitted over only one generation (from generation1 to generation2) are not transmitted again (from generation2 to generation3): therefore, they can only be causally responsible for short-term effects lasting during a limited evolutionary stage (from generation1 to generation2), but not across the long-term evolutionary time. Moreover, as

\footnotetext{
${ }^{25}$ Hereditary factors transmitted over only one generation and over many generations can vary depending on the species considered and the particular contingent history of the organisms concerned (e.g., there are big differences between metazoans and other living beings like bacteria as to the transmission of hereditary factors over time). It is also worth to note that hereditary factors are different with regard to the unit of evolution considered (populations of genes, cells, organisms, etc.).
} 
such factors are transmitted over only one generation, they cannot be subjected to several events of selection and/or drift, so they can rarely lead up to significant evolutionary effects (e.g. adaptations) because they die out after only one generation.

Let us now consider the second category of hereditary factors I distinguished above, that is the set of developmental factors transmitted over many generations. In contrast to factors transmitted over only one generation, hereditary factors belonging to this second category can have a causal responsibility in both short-term and long-term evolutionary effects. In virtue of the fact that they can be passed on from parents to offspring in many successive generations, they can be subjected to several events of selection and/or drift over time, so they can lead up to major long-term consequences in evolution: in particular, in the case of cumulative selection, they can lead up to adaptations ${ }^{26}$.

On the basis of the distinction of these two categories of developmental factors with respect of their evolutionary potential, I advance my criticisms against Griffiths and Gray's "causal parity" thesis in the case of evolution. I argue that all genetic and extra-genetic hereditary factors are not on a par as causes of evolution, and so are not equally important in order to explain the evolutionary process, because some factors (developmental factors transmitted over many generations) can produce more significant long-term evolutionary results than others (developmental factors transmitted over only one generation). For instance, let us consider inheritance in mammals (e.g., elephants) and investigate the respective evolutionary potential of some factor transmitted over many generations (e.g., some allele) and of some factor transmitted over only one generation (e.g., a methylation pattern) ${ }^{27}$.

\footnotetext{
${ }^{26}$ As Sterelny et al (1996: 400) says: "If selection is to explain major adaptation it must be cumulative. Innovation is the result of a long sequence of selective episodes rather than one".

${ }^{27}$ It is worth to notice that methylation patterns can also be transmitted over more than one generation: it depends on the species and on the contingent history of the organisms considered. For instance, methylation marks can be transmitted over many generations in unicellular organisms and in pluricellular organisms with
} 
First, let us deal with genetic inheritance and its potential evolutionary consequences. Let us imagine a situation in which two allelic forms ( $A$ and $a$ ) of a gene involved in the expression of a phenotypic trait $\mathrm{P}$ (e.g. ear size) are present at locus $\mathrm{L}$ and have the same frequency $(50 \% A$ and $50 \% a)$ in a population of generation1-mammals (e.g., elephants). Furthermore, let us assume that, during generation1, environmental conditions change (e.g., there is a sudden increase of temperature) and that, in such a new situation, individuals with the $A$ allele turn out to be fitter than individuals with the $a$ allele (e.g., because the $A$ allele contributes to the production of big ears, which are better than little ones to regulate body temperature). Thus, generation1-organisms (elephants) with the $A$ allele are positively selected, the $A$ allele is transmitted to the next generation, and so its phenotypic effect (the trait "big ear size") too: in generation2-population, the $A$ allele frequency increases (the $a$ allele frequency decreases). If generation2-individuals with the $A$ allele turn out again to be fitter than individuals with the $a$ allele (e.g., because the temperature remains high, or because it increases further), they are positively selected again, the $A$ allele is transmitted to the next generation and, as a result, the phenotypic trait P ("big ear size") too: in generation3population, the frequency of $A$ further increases (the frequency of $a$ further decreases). We can imagine that the phenotypic trait P ("big ear size") is subjected to many events of selection in successive generation (i.e., cumulative selection), so that the $A$ allele and the trait P ("big ear size") are transmitted, more or less reliably, over the long-term evolutionary time: in this manner, the $A$ allele can come to fix in the population and lead up to a long-term evolutionary effect (an adaptation, e.g., a certain big ear size).

Let us now consider the inheritance of methylation marks, which in mammals are rarely transmitted from parents to offspring because they are usually erased in germ-line cells.

asexual reproduction; on the contrary, in mammals they are rarely transmitted over more than one generation and, more often, they are not transmitted at all. See Jablonka and Lamb 2005. 
Anyway, it can happen that they are transmitted over one generation through the process biologists call "genomic imprinting" 28 . So, let us at least assume that some methylation marks are transmitted over one generation (from generation1 to generation2). Contrary to the case of the $A$ allele described above, the methylation marks cannot be subjected to several events of selection and so cannot lead to any major evolutionary effect (an adaptation): in fact, even though they can play a causal role in the differential survival and reproduction among generation1-organisms (mammals) and they can be passed on from one generation to the next (from generation1 to generation2), then they are erased during the embryonic development of generation2-offspring and so they cannot be further transmitted (from generation2 to generation3). Therefore, as Jablonka and Lamb claim (2005: 140) "such constantly changing epigenetic marks are not likely to be the raw material for adaptive evolution".

Finally, let us examine a situation concerning both genetic and epigenetic hereditary factors, in particular in which epigenetic changes influence the selection of genetic variation (i.e., genetic assimilation) ${ }^{29}$. Let us imagine that the genome of some organisms (e.g., mammals) in a generation1-population is characterized by some methylation marks inhibiting the expression of some gene $\mathrm{G}$ at locus L: genetic variation can accumulate at the silenced locus L without being expressed, and the effect is always some phenotype I call P1. Let us further imagine that some environmental stress (e.g., a heat-shock) induces a change of the methylation marks so that the hidden genetic variation at the silenced genetic locus $\mathrm{L}$ is

\footnotetext{
${ }^{28}$ During the egg and the sperm production, chromosomes acquire respectively a set of maternal chromatin marks and a different set of paternal chromatin marks. However, such chromatin marks are intrinsically transient because they are erased when one chromosome passes from one sex to another and new sex-specific marks are established. See Jablonka and Lamb 2005: 139-140, 254-258.

${ }^{29}$ Genetic assimilation is a process whereby phenotypic variation induced by environmental stimuli becomes genetically fixed in a population: in this manner, phenotypic variation no longer requires the environmental signal for expression.
} 
revealed: as a result of the induced epigenetic changes, the gene $\mathrm{G}$ is expressed and the effect is a different phenotype I call P2. The environmentally induced phenotypic variation (P2) can be reproduced in the next generation (generation2) if the induced epigenetic changes are transmitted from parents to offspring (from generation1 to generation2) ${ }^{30}$. Or, if the environmental stimulus (the heat-shock) lasts in generation2, it can induce again the same change of methylation marks, and so the phenotype P2. However, if we assume that methylation marks are transmitted over only one generation and we imagine that the environmental stimulus wears off after a few generations, we can conclude that, in this case, the phenotypic variation P2 can just be a short-term evolutionary effect because it disappears from the population after a few generations (when methylation marks are erased or when the environmental stimulus wears off). On the contrary, if the hidden genetic variation expressed in stressful environmental conditions reveals to be advantageous (i.e., if the induced phenotype P2 reveals to be advantageous), it can be positively selected, transmitted from parents to offspring, be subjected to further selective events over many generations, and finally fix in the population: the phenotypic variant P2 come to be expressed even when epigenetic marks are erased and without any inducing environmental stimulus. Only in this case (i.e., in virtue of the transmission of some factor over many generations, which in this case is the gene G) the phenotype P2 can evolve and turn out to be a long-term significant evolutionary effect (e.g., an adaptation).

The three situations I have just described above, in particular the last one (i.e., the process of genetic assimilation), clearly show the difference between, on the one hand, the evolutionary potential of factors transmitted over only one generation (e.g., methylation marks), which can only have short-term effects limited to the evolutionary step they are

\footnotetext{
${ }^{30}$ As I said above, this is not always the case in pluricellular organisms with sexual reproduction, and in particular in the case of mammals I consider here.
} 
passed on from one generation to the next; on the other hand, the evolutionary potential of factors transmitted over many generations (e.g., genes), which can be subjected to cumulative events of selection and/or drift and so can produce long-term effects in evolution. Therefore, against Griffths and Gray's "causal parity" thesis in evolution, I maintain that developmental factors transmitted over many generations are more important as evolutionary causes and as explanatory factors of evolution in comparison with factors transmitted over only one generation: the potential evolutionary effects of the former are more significant over time than the potential evolutionary scope of the latter.

I conclude by underlining both the aspects of Griffiths and Gray's perspective on evolution I agree with and the aspects I criticize. I am sympathetic with their criticisms against the traditional characterization of genes (or DNA sequences) as the only transmitted master molecules carrying the program for development: genes might have no privileged causal responsibility in evolution. More precisely, the major value of Griffiths and Gray's view of evolution is in that they underline the important causal contribution of extra-genetic hereditary factors to the differential survival and reproduction among organisms. (i.e., to selection, and so to the evolutionary process). Nevertheless, their perspective on extra-genetic inheritance turns out to be too radical and not useful for experimental research because of its holistic character: even though the "heredity holism" (Griesemer et al 2005: 526) Griffiths and Gray advance is valuable, in some respect, because it keeps always in view the contextdependency of every factor on the others, it risks losing sight of their differences. Actually, in order to contrast the conventional gene-centric view of evolution, Griffiths and Gray tend to consider all hereditary factors as equally important in evolution, and so ignore some distinctions (e.g., the difference I showed above between the evolutionary potential of factors transmitted over only one generation and over many generations) which are really important to understand the respective causal and explanatory role of hereditary factors as to evolution. 
Thus, with my present argument, I do not intend to maintain that developmental factors transmitted over only one generation, and also factors not transmitted at all, cannot have a causal role in evolution and are not relevant to explain the evolutionary process. On the contrary, as I stressed above, if such a factor partly determines the survival and the reproduction of some organism rather than another, it definitely has a causal role in evolution. However, I argue that Griffiths and Gray cannot put such factors on a par with developmental factors transmitted over many generations as to their causal role in evolution: indeed, the potential evolutionary effects due to factors transmitted over only one generation usually have a short-term scope, limited to a few generations, and rarely contribute to more significant long-term consequences in evolution.

\section{Conclusion}

In this paper, I put forward three main criticisms of Griffiths and Gray's reworking of the concept of inheritance and evolution.

First, their concept of expanded and diffused inheritance is certainly richer and more complete than other accounts of inheritance limited to a small set of hereditary factors in additive interaction; nevertheless, because of its holistic character, its translation into novel theoretical models and into alternative analytical tools for researches on biological inheritance reveals to be a problematic task.

Second, by looking at the mechanisms of inheritance and their impact on evolutionary dynamics, Griffiths and Gray's holistic account of inheritance reveals to be less biologically realistic than other approaches because of its refusal to distinguish multiple systems of inheritance; on the contrary, such a distinction has a central role in biology, not only as a heuristic tool, but also as a more realistic representation of evolutionary phenomena. 
Third, Griffiths and Gray's evolutionary "causal parity" thesis is not sufficiently warranted because it does not take into account the important difference between developmental factors transmitted over only one and developmental factors transmitted over many generations as regard their causal role in evolution. 


\section{Acknowledgments}

Many thanks to Marie-Claude Lorne, Anouk Barberousse, Thomas Pradeu, Susan Oyama, Michael Trestman, Roberta Millstein, James Griesemer, Vadim Keyser, Ayelet Shavit, Eric Bapteste, Pierre-Alain Braillard, Michel Morange, Arnaud Pocheville.

\section{$\underline{\text { References }}$}

BARRANGOU, R. et al (2007): 'CRISPR Provides Acquired Resistance Against Viruses in Prokatyotes', Science, 315, 1709-1712.

DAWKINS, R. (1976): The Selfish Gene, Oxford University Press, Oxford.

DAWKINS, R. (1982): The Extended Phenotype, Freeman, Oxford.

DURHAM, W.H. (1991): Coevolution: Genes, Culture, and Human Diversity, Stanford University Press, Stanford.

GODDE, J.S. and BICKERTON, A. (2006): 'The Repetitive DNA Elements Called CRISPRs and Their Associated Genes: Evidence of Horizontal Transfer Among Prokaryotes', Journal of Molecular Evolution, 62, 718-729.

GRAY, R.D. (1992): 'Death of the gene: Developmental systems strike back', In Griffiths, PE (ed) (1992) Trees of life, Dordrecht: Kluwer,165-210.

GRAY, R.D. (2001): 'Selfish genes or developmental systems?', In Thinking about evolution: historical, Philosophical and Political Perspectives: Festschrift for Richard Lewontin, Singh R., Krimbas K., Paul D. and Beatty J., Cambridge University Press, Cambridge GRIFFITHS, P.E. and GRAY, R.D. (1994): 'Developmental Systems and Evolutionary Explanation', The Journal of Philosophy 91, 6, 277-304

GRIFFITHS, P.E. and GRAY, R.D. (1997): ‘The Replicator II: Judgement Day’, Biology and Philosophy, 12, 4, 471-492. 
GRIFFITHS, P.E. and GRAY, R.D. (2004): 'The Developmental Systems Perspective. Organism-Environment Systems as Units of Development and Evolution', In M. Pigliucci and K. Preston (eds), Phenotypic Integration - Studying the Ecology and the Evolution of Complex Phenotypes, Oxford University Press, Oxford.

GRIFFITHS, P.E. and GRAY, R.D. (2005): 'Discussion: Three Ways to Misunderstand developmental systems theory', Biology and Philosophy, 20, 417-425.

Griffiths, P.E. and Knight, R.D. (1998): 'What is the Developmental Challenge?', Philosophy of Science, 65, 2, 253-258.

HULL, D. and WILKINS, J.S. (2005): 'Replication', The Stanford Encyclopedia of Philosophy (Fall 2008 Edition), Edward N. Zalta(ed.), URL = $<$ http://plato.stanford.edu/archives/win2008/entries/replication/>.

JABLONKA, E. and LAMB, M. (2005): Evolution in Four Dimensions. Genetic, Epigenetic, Behavioral, and Symbolic Variation in the History of Life, MIT Press, Cambridge MA.

KITCHER, P. (2001): 'Battling the Undead: How (and How Not) to Resist Genetic Determinism', in Singh, RS, Krimbas, CB, Paul, DB and Beatty, J, Thinking About Evolution. Historical, Philosophical, and Political Perspectives, volume two, Cambridge University Press, Cambridge.

LALAND, K.N., ODLING-SMEE, F.J., FELDMAN, M.W. (2003): Niche Construction : The Neglected Process in Evolution, Princeton University Press.

MAKAROVA, K.S. et al (2002): 'A DNA repair system specific for thermophilic Archaea and bacteria predicted by genomic context analysis, Nucleic Acids Research, 30, 482-496.

OYAMA, S. (1985): The Ontogeny of Information: Developmental Systems and Evolution, Cambridge University Press, Cambridge.

OYAMA, S. (2000a): Evolution's Eye, Duke University Press, Durham 
OYAMA, S. (2000b): Causal Democracy and Causal Contributions in Developmental Systems Theory, Proceedings PSA, 67, S332-347

OYAMA, S., GRIFFITHS, P.E., GRAY, R.D. (eds) (2001): Cycles of Contingency. Developmental Systems and Evolution, Cambridge, MIT Press

STERELNY, K., DICKINSON, M., SMITH, K. (1996): 'The extended replicator', Biology and Philosophy, 11, 3, 377-403.

WATERS, K. (2006): 'A Pluralist Interpretation of Gene-Centered Biology', Minnesota Studies in the Philosophy of Science Series, 19, 190-214. 